\title{
Concilier vie professionnelle et aide informelle à un parent âgé
}

Un défi des 50-64 ans en Europe

Combining professional life and support given to an ageing parent: a challenge for persons aged 50-64 in Europe

\section{Malorie Peyrache et Jim Ogg}

\section{OpenEdition}

\section{Journals}

Édition électronique

URL : http://journals.openedition.org/ress/3841

DOI : 10.4000/ress.3841

ISBN : $1663-4446$

ISSN : 1663-4446

Éditeur

Librairie Droz

Édition imprimée

Date de publication : 15 mai 2017

Pagination : $97-125$

ISSN : 0048-8046

Référence électronique

Malorie Peyrache et Jim Ogg, " Concilier vie professionnelle et aide informelle à un parent âgé », Revue européenne des sciences sociales [En ligne], 55-1 | 2017, mis en ligne le 15 mai 2020, consulté le 19 janvier 2021. URL : http://journals.openedition.org/ress/3841 ; DOI : https://doi.org/10.4000/ress. 3841 


\title{
CONCILIER VIE PROFESSIONNELLE ET AIDE INFORMELLE À UN PARENT ÂGÉ \\ UN DÉFI DES 50-64 ANS EN EUROPE
}

MALORIE PEYRACHE \& JIM OGG

Paris, CNAV, URV

malorie.peyrache@cnav.fr / jim.ogg@cnav.fr

\begin{abstract}
Résumé. Cet article met en perspective le taux d'emploi et d'aide informelle parmi les 50-64 ans ayant au moins un parent vivant dans quatre pays européens, sur les deux dernières vagues disponibles l'enquête «Share» 2010 et 2012 (Vagues 4 et 5). En effet, l'allongement de l'espérance de vie et le recul de l'âge de départ à la retraite questionnent l'articulation entre vie professionnelle et aide informelle à un parent âgé. En outre, l'engagement de plus en plus important des femmes sur les marchés du travail en Europe alors que celles-ci sont les principales aidantes des parents âgés fait craindre une pression d'autant plus forte sur les systèmes de protection sociale. La perception de l'état de santé du parent joue un rôle primordial sur l'aide apportée à celui-ci, et l'intensité de cette aide joue également un rôle important dans la probabilité d'être en emploi.
\end{abstract}

Mots-clés: aide informelle, disparités européennes, emplois, séniors, solidarité intergénérationnelle.

\begin{abstract}
This paper puts into perspective employment rates and help given to parents by adults aged 50-64 years with at least one parent alive in four European countries. The data are from the Waves 4 and 5 of the "Share" survey collected in 2010 and 2012. Increasing life expectancy and policies directed towards active ageing and postponing retirement bring into question the articulation between the end of professional life and support given to an ageing parent. The continued increase of older women in the labour market is placing pressure on informal systems of care for older people. The perception of the health status of a parent is an important factor influencing the provision of help and the intensity of help interacts with the probability of being in paid employment.
\end{abstract}

Keywords: European differences, informal help, intergenerational solidarity, paid work, seniors. 
INTRODUCTION

Dans un contexte d'une durée de vie de plus en plus longue qui n’a pas été accompagnée par une baisse de la durée de vie sans incapacité pendant la vieillesse (Gill et al., 20Io), mais également d'un recul de l'âge du départ à la retraite, l'aide informelle auprès des personnes âgées apportée par leurs enfants s'effectue de plus en plus par des descendants qui sont encore en activité. La place centrale donnée à l'aide informelle par les politiques publiques rencontre une difficulté majeure, dans la mesure où les enfants des parents âgés fragilisés ou dépendants doivent rester plus longtemps sur le marché de travail avant de prendre leur retraite. En effet, le Conseil européen de Stockholm en 200I a «convenu de se fixer pour l'horizon 2010 l'objectif de porter à $50 \%$ le taux d'emploi moyen dans l'Union européenne pour la catégorie d'âge, hommes et femmes confondus, de 55 à 64 ans ». Ce même objectif d’amener le taux d'emploi à $50 \%$ pour les séniors a été par la suite renouvelé par le programme communautaire de Lisbonne en 2005, même si certains pays européens ne l'ont pas encore atteint. Depuis 20Io, la politique européenne en termes d'emploi s'est accentuée autour de la notion de «vieillissement actif», un terme générique qui désigne entre autres, la nécessité pour les séniors de travailler plus longtemps afin d'équilibrer les systèmes de retraite. En conséquence, l'incitation à travailler plus longtemps peut se heurter aux politiques de soins de longue durée qui privilégient l’aide informelle.

Dans ce contexte, de plus en plus de personnes peuvent être confrontées à un choix : rester sur le marché du travail pour obtenir une retraite optimale ou le quitter pour consacrer le temps nécessaire à l'accompagnement et à l'aide d'un parent âgé. La question de l'articulation entre vie familiale et vie professionnelle, qui est à la fois un objet de recherche et un enjeu politique de premier ordre dans le cadre des politiques d'égalité entre femmes et hommes, est toujours d'actualité (Le Bihan Youinou et Martin, 2006). En ce qui concerne l'interaction de ce choix avec les politiques en vigueur relatives au soin de longue durée, plusieurs études sur les effets de «crowding out» ou «crowding in» ont été réalisées (Kemper, 1992; Roe et al., 200I ; Bonsang, 2009; Litwin et Attias-Donfut, 2009), mais la plupart de ces travaux ont mis en évidence une complémentarité entre l'aide formelle et informelle, en soulignant donc le fait qu'elles ne se substituaient pas entre elles 
(Attias-Donfut et Ogg, 2009). Par rapport aux choix auxquels les individus sont confrontés, des coûts plus ou moins directs peuvent apparaître pour les enfants accordant du temps à leurs parents âgés par le biais d’aide informelle. Ces coûts surgissent, par exemple, quand une heure est utilisée pour l'apport de soin informel au lieu de l'être sur le marché du travail. Les enfants fournissant des soins informels à leurs parents âgés peuvent donc subir des effets négatifs accumulés au fil du temps sur leur situation professionnelle se traduisant par une dégradation de leur revenu (Bolin et al., 2008). Les travaux de Bérengère Davin et al. (2009) proposant une valorisation monétaire du soutien familial aux personnes âgées dépendantes illustrent le poids économique considérable du soutien familial.

Ces effets indésirables peuvent être différents selon les dispositions institutionnelles spécifiques à chaque pays, lesquels peuvent également inciter à la mise en place d'une grande diversité de modèles d'aides en Europe. Inversement, ces mêmes différences institutionnelles peuvent être le reflet des divergences culturelles en matière de normes, de traditions et de préférences. Les différences de normes concernant le soin de longue durée ont tendance à suivre en Europe un axe Nord-Sud (Kohli et al., 2005). Les pays scandinaves sont ainsi cités pour avoir les relations familiales parent-enfant «les plus faibles», les pays méditerranéens «les plus fortes», tandis que les pays continentaux se situent dans une situation dite intermédiaire (Hank, 2007). Or, dans le contexte actuel d'accélération des transformations sociales et économiques, il convient de s'interroger sur la persistance de ces tendances, et l'existence d'un gradient Nord-Sud dans les effets du soin informel sur le marché du travail.

Une première étape doit consister à mettre en parallèle le taux d'emploi des séniors en âge actif, en sachant que depuis la crise économique de 2008, les taux de participation des séniors sur le marché de travail ont connu des évolutions différenciées'. Pour ce faire, quatre pays européens feront l'objet de l'étude - la Suède, l’Allemagne, la France, et l'Espagne - choisis de manière à permettre la comparaison d'un pays d'Europe du Nord (Suède), un pays

I II faut rappeler que les âges légaux et effectifs de départ en retraite varient considérablement selon les pays (CCSS, 2010). 
d'Europe du Sud (Espagne), et de deux pays d'Europe continentale à travers le duo franco-allemand. Chacun de ces quatre pays représente des modèles traditionnellement différents par rapport à l'organisation des systèmes de «welfare», bien que ces différences soient devenues plus complexes que le modèle d’Esping-Andersen (1990) ne les avait décrites il y a plus de vingt ans.

En Suède, les dépenses publiques de prise en charge de la dépendance atteignaient 3,6\% du PIB en 2007 (Centre d'analyse stratégique), part qui restait inchangée si l'on prenait en compte en compte les dépenses privées (OECD Health Data 2010) ${ }^{2}$. La Suède, qui fait partie des pays du Nord précurseurs à avoir légiféré sur le sujet, a mis en place un dispositif spécifique de protection sociale contre la perte d'autonomie dès 1964 suite à la suppression en 1956 des obligations juridiques d'assistance des enfants envers leurs parents. Ce modèle souvent qualifié de «social-démocrate» ou «nordique » d'inspiration beveridgienne, s'apparente à une couverture sociale universelle financée par l'impôt et qui favorise le placement en institution des personnes dépendantes. Depuis une dizaine d’années, la part de la population des séniors actifs sur le marché du travail en Suède a continué d'augmenter, et la Suède reste l'un des pays de l'Europe avec des taux de séniors actifs parmi les plus élevés. Si la Suède est connue pour son modèle universaliste d'aide aux personnes âgées et pour une forte implication de l'Etat dans la provision des soins, ce modèle est cependant de plus en plus remis en question. Depuis les années 1990, l'introduction des principes du marché dans le système fait de plus en plus porter la responsabilité de l'aide sur les familles, de sorte que l'aide d'un enfant adulte à un parent âgé fragilisé ou dépendant est aujourd'hui répandue (Ulmanen et Szebehely, 20I5).

D’après le Centre d’analyse stratégique, les dépenses publiques de prise en charge de la dépendance atteignaient 0,9\% du PIB en Allemagne en 2007, cette part passait à I, $3 \%$ du PIB si l'on prenait également en compte les dépenses privées (OECD Health Data, 20ı). En ce qui concerne les politiques de soins de longue durée, l’Allemagne a privilégié la famille et plus précisément les femmes jusqu’à

2 Les chiffres du Centre d'analyse stratégique et de l'OECD Health Data sont issus du Rapport d'information n³667 de l'Assemblée Nationale sur «la prise en charge de la dépendance en Europe» déposé par la Commission des affaires européennes, Juillet 2011 . 
l'introduction d'un dispositif en 1994 instaurant un régime d'assurance soins de longue durée (Pflegeversicherungsgesetz) et la création d'une nouvelle branche de la sécurité sociale. Le modèle allemand, de type bismarckien et sa logique assurantielle, est conçu comme une couverture spécifique financée par les cotisations sociales. Les aidants informels peuvent bénéficier d'une compensation partielle grâce au système d’assurance. Cependant, le système reste très fragmenté, avec une disparité des services entre les régions et des formes hybrides de soins combinant les principes du marché à de l’aide informelle, formelle, privée et associative. En Allemagne, le taux d'emploi des femmes sur le marché du travail est resté faible jusquaux années 1990. Pendant la période 2004 à 2010, la tendance est à l’accroissement de la part des femmes actives sur le marché du travail, le taux d'emploi passant de 34\% à 6I,2 \% pour les femmes âgées de 55 à 64 ans (Eurostat, Open Data).

En France, les dépenses publiques de prise en charge de la dépendance atteignaient I,4\% du PIB en 2007 (Centre d’analyse stratégique), et I,7\% si l'on prend également en compte les dépenses privées (OECD Health Data, 20I0). Historiquement, comme en Allemagne et en Espagne, l'aide aux personnes âgées reste principalement une obligation de la famille, mais depuis une vingtaine d’années plusieurs dispositifs ont été introduits pour soutenir l'aide familiale, notamment l'Allocation Personnalisée d'Autonomie (APA) en 2002. Le taux de participation au marché de travail des personnes âgées de 55 à 64 ans a augmenté de 36,I \% à 45,7\% pendant la période de 2002 à 20ı2. Ce taux relativement bas par rapport à d'autres pays européens, est lié à un âge de départ effectif à la retraite plus précoce en France que dans le reste de l'Europe. Cependant, les taux élevés de chômage des séniors perdurent et freinent l'effet des mesures et des incitations visant à prolonger la vie active. Pendant la période 2003-2013, le taux de chômage pour les hommes âgés de 55 à 59 ans a augmenté de $4,7 \%$ à $8 \%$ et également celui des femmes aux mêmes âges, passant celui-ci de 4,4\% à 6,8\% (Minni, 20I5).

En Espagne, les dépenses publiques de prise en charge de la dépendance atteignaient $0,5 \%$ du PIB en 2007 (Centre d’analyse stratégique), alors que cette part atteignait $0,8 \%$ si l'on prenait également en compte les dépenses privées (OECD Health Data, 20I0). Dès 2007, l'Espagne a mis en place un dispositif de prise en charge universel de la dépendance couvrant sans disposition d'âge la perte 
d'autonomie. Si le taux d'emploi des femmes en Espagne a augmenté constamment depuis les années 1970, la majorité des femmes séniors actives travaille toujours à mi-temps. Malgré l'introduction des dispositifs qui visent à réconcilier la vie privée et publique, l’aide apportée par la famille à des parents fragiles ou dépendants semble donc rester importante, bien que les classes moyennes fassent de plus en plus appel au marché des aidants immigrés (Guner et al., 20I4).

Une des hypothèses déjà soulevée serait que les effets potentiellement indésirables du soin informel sur le taux d'emploi des enfants aidants soient moins marqués dans les pays où la prise en charge des personnes âgées dépendantes repose en majeure partie sur des systèmes de solidarités publiques (Fontaine, 2009). En Suède par exemple, près de 2,6\% du PIB était consacré aux dépenses de soins de longue durée en 2004 et près de $7 \%$ des plus de 65 ans vivaient en institution au début des années 1990. À l'inverse, dans les pays d'Europe du Sud où les dispositifs publics de prise en charge sont moins importants, à l'instar de l'Espagne qui consacrait en 2004 près de $0,3 \%$ du PIB aux soins de longue durée alors que moins de $3 \%$ de la population des plus de 65 ans vivaient en institution (Bolin et al., 2008).

Le but de cette étude est donc de mieux connaître l'effet que peut avoir le taux d’aide informelle apportée par les séniors à leurs parents sur leur situation professionnelle, tout en analysant les disparités et les évolutions de ces taux. Quand bien même il doit être tenu compte de la double relation causale entre le fait d'être en emploi et le fait d'apporter de l'aide à un parent âgé, la question demeure de savoir si l'apport d’aide informelle des séniors est compatible ou non avec une politique visant à prolonger l'activité des séniors; et s’il ne faut pas craindre une diminution de cette aide en cas d'augmentation du taux d'emploi de ces aidants séniors.

Après avoir présenté l'enquête «Share» et la méthodologie utilisée pour notre analyse, nous commenterons les résultats des taux d'emploi et le lien entre le fait d'avoir un parent vivant et la participation sur le marché de l'emploi. Ensuite, nous analyserons les résultats quant aux taux d’aide informelle apportée par des séniors à leurs parents âgés ainsi que les disparités et évolutions de ces apports. Pour finir, nous nous pencherons sur les différents modèles réalisés qui déterminent les probabilités, respectivement, d'être en emploi, d’apporter de l'aide à la mère et de l'aide au père. 


\section{PRÉSENTATION DE L'ENQUÊTE «SHARE» ET ÉLÉMENTS MÉTHODOLOGIQUES}

L'enquête «Share » (Survey on Health, Ageing and Retirement in Europe) permet de disposer de données pluridisciplinaires et internationales sur des individus âgés de 50 ans et plus. L'objectif est de comparer le taux d'emploi des personnes âgées de 50 à 64 ans, à partir de la vague 4 (2010-20II) et de la vague 5 (20I2-20I3), selon que la personne a un ou deux parents vivants et apporte ou non un soutien informel. La limite de 65 ans a été choisie, afin de cibler au maximum les personnes en âge actif. Cette limite d'âge a bien entendu des conséquences sur le taux d'emploi selon les pays, l'âge légal de départ à la retraite n'étant pas commun à tous les pays européens. Les données de l'enquête «Share» permettent d'examiner les différents aspects sociaux du vieillissement et plus précisément, dans notre étude, le lien entre taux d'emploi et soin informel selon le sexe et l'âge de l'enquêté ainsi que son évolution dans le temps grâce aux différentes vagues de l'enquête, et ce dans chacun des quatre pays choisis (la Suède, l’Allemagne, la France et l’Espagne). L'étude comparative de nos deux vagues pourrait éventuellement permettre de déceler une tendance, une stabilisation ou un décrochage de l'aide apportée à un parent âgé.

Dans cette analyse, nous nous concentrons à la fois sur le taux d'emploi et sur l'aide informelle apportée aux parents biologiques des individus âgés entre 50 et 64 ans ayant au moins un parent en vie. Notre échantillon contient donc i 857 individus en vague 4 et 469 I en vague 5. Entre les deux vagues, $84 \mathrm{I}$ individus sont sortis de l'observation tandis que les I o 6 restants sont également présents dans la cinquième et dernière vague. Pour compléter l'effectif de cette vague 5, 3675 individus âgés de 50 ans et plus sont entrés dans le champ d'observation soit $73 \%$ de notre sous-échantillon, suite à un «rafraîchissement du panel d'enquêtés. Ces effectifs ne sont pas pondérés, cependant pour obtenir et analyser nos résultats nous les affinerons en utilisant les données pondérées de ces sous-échantillons. 
Tableau I. Effectifs et filtres appliqués aux sous-échantillons

\begin{tabular}{|c|c|c|}
\hline EFFECTIFS & VAGUE 4 & VAGUE 5 \\
\hline Au départ & 83289 & 94118 \\
\hline $\begin{array}{l}\text { Après filtre } \\
\text { des répondants à l'interview }\end{array}$ & 58202 & 66246 \\
\hline $\begin{array}{l}\text { Après filtre } \\
\text { «4 pays sélectionnés» }\end{array}$ & 13169 & 21530 \\
\hline $\begin{array}{l}\text { Après filtre } \\
\text { «âge compris entre } 50 \text { et } 64 \text { ans » }\end{array}$ & 5571 & 9154 \\
\hline $\begin{array}{l}\text { Après filtre } \\
\text { «au moins un parent vivant» }\end{array}$ & 1857 & 4691 \\
\hline
\end{tabular}

Note de lecture: avant l'application des filtres de notre analyse, il y avait 83289 individus présents en vague 4. Champs: effectifs non-pondérés d'individus des vagues 4 et 5 de l'enquête «Share».

Source: enquête «Share», 1857 individus en 2010 et 4691 individus en 2012.

Dans l'enquête «Share », l'aide informelle est caractérisée selon les réponses qui correspondent à l'aide personnellement donnée au cours des 12 derniers mois à une personne, membre de la famille ami ou voisin, extérieure ou au sein du ménage. Nous considérerons par la suite comme aidants, l'ensemble des enfants ne cohabitant pas avec leur parent âgé mais déclarant lui apporter une aide dans les soins personnels, dans les activités domestiques ou administratives. Nous considérons également comme aidants, dans tous nos résultats, l'ensemble des enfants corésidents dans la mesure où il est plus que probable qu'ils apportent d'une manière ou d'une autre, une aide quasi quotidienne à leur parent âgé. En effet, nous faisons l'hypothèse que les enfants corésidents avec leurs parents âgés aident quotidiennement ces derniers de manière consciente ou non, que ce soit dans le partage des tâches domestiques et administratives, ou d'une autre façon. Le fait de vivre dans le même ménage rend invisibles les activités quotidiennes considérées comme de l'aide informelle à l'extérieur du ménage (Ogg et Renaut, 2006). La cohabitation semble par ailleurs une réponse à la dépendance d'un parent âgé sans conjoint, en particulier dans les pays du sud de l'Europe (Fontaine et al., 2007). Aussi, la corésidence intergénérationnelle pourrait constituer une réponse à l'entrée en dépendance 
du parent, ce qui justifie notre choix de considérer les corésidents comme des aidants quotidiens envers leurs parents âgés.

Dans l'apport d’aide informelle à un individu extérieur au ménage de l'enquêté, les types d'aide informelle comptabilisés sont: les soins personnels (se laver, manger, se coucher ou se lever, aller aux toilettes, se vêtir ou se dévêtir), les aides ménagères (petits travaux tels que du bricolage, jardinage, déplacements, courses), et les aides administratives (remplir des formulaires, régler des questions financières ou juridiques).

Pour calculer le taux d'emploi et le taux d’activité, nous avons fondé notre analyse sur les modalités suivantes: retraité(e), salarié(e) ou travaillant à son propre compte incluant ou non le travail pour une affaire familiale, chômeur(se) et en recherche d'emploi, invalide ou en congé de longue maladie, homme ou femme au foyer, autre (rentier, étudiant, bénévole), ne sait pas, et refus. Pour le calcul du taux d'emploi, nous rapportons seulement le nombre d'individus ayant déclaré être salariés ou travaillant à leur propre compte sur le nombre total d'individus dans notre échantillon. Concernant le taux d'activité, nous faisons la somme du nombre d'individus ayant déclaré être salariés ou à leur propre compte avec ceux ayant déclaré être chômeurs et en recherche d'emploi, pour ensuite rapporter cette somme au nombre total d'individus dans notre échantillon.

Plusieurs variables sociodémographiques sont utilisées dans notre analyse. Ces variables vont en premier lieu concerner l'état de santé perçu par l'enquêté concernant sa mère et son père. Le statut matrimonial de l'enquêté va également être pris en compte, ainsi que la corésidence avec la mère et le père.

Nous constaterons des écarts de taux d'emploi selon le sexe et le pays ainsi que l'évolution dans le temps des individus âgés entre 50 et 64 ans ayant au moins un parent vivant. Ce faisant nous porterons notre attention sur l'apport ou non d’aide informelle à un parent âgé, au moyen de statistiques descriptives, ainsi que des taux standardisés (cf. Encadré I), mais également de techniques de régression. 


\section{Encadré I. Méthode de standardisation directe}

L'objectif de la standardisation consiste à éliminer l'effet des différences entre populations à comparer pour la variable considérée (ici l'âge): dans ces conditions, on pourra admettre que, si on constate une différence entre les taux obtenus, cette différence n'est pas le fait de l'âge.

On souhaite comparer des populations entre elles, ici celles de la vague 4 et de la vague 5 par pays. On utilise alors la standardisation directe qui consiste à définir une population de référence, ou population type, et à faire en sorte que chacune des populations à comparer ait une structure d'âge identique à celle de cette population de référence. Les taux que l'on calcule alors pour chacune des populations sont appelés taux standardisés sur l'âge car dans notre cas, c'est la variable âge qui est prise en compte.

Nous souhaitons par exemple comparer le taux d'emploi en Allemagne de la vague 4 et celui de la vague 5 de l'enquête «Share» en standardisant sur l'âge. La population de référence utilisée va être celle de notre sous-échantillon en Allemagne en vague 4 . On applique pour ce faire les taux d'emploi par âge détaillé de notre population en vague 5 à la structure par âge de celle de la vague 4. Le taux global standardisé qui en ressort décrit alors ce que serait le taux d'emploi de notre population en vague 5 si celle-ci avait eu la même structure par âge qu'en vague 4. En neutralisant l'effet de l'âge dans le taux d'emploi, cela nous permet de mieux pouvoir les comparer.

Tableau 2. Caractéristiques sociodémographiques de nos sous-échantillons

\begin{tabular}{|c|c|c|c|c|c|c|c|c|c|c|}
\hline & \multicolumn{5}{|c|}{ VAGUE 4} & \multicolumn{5}{|c|}{ VAGUE 5} \\
\hline & 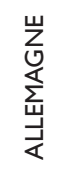 & 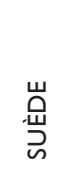 & $\begin{array}{l}\text { U } \\
\text { U } \\
\vdots \\
\text { 岀 }\end{array}$ & $\begin{array}{l}\text { U } \\
\underset{\mathbb{u}}{\mathrm{u}} \\
\text { 足 }\end{array}$ & 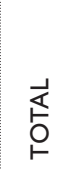 & 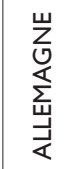 & 闿 & 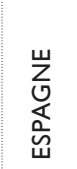 & 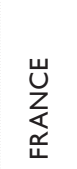 & $\begin{array}{l}\vec{\leftarrow} \\
\stackrel{5}{O} \\
\vdash\end{array}$ \\
\hline Filtre âge «50-64 ans» & 625 & 608 & 1498 & 2840 & 5571 & 2944 & 1586 & 2670 & 1954 & 9154 \\
\hline Filtre «au moins un parent vivant» & 185 & 213 & 296 & 1163 & 1857 & 1543 & 784 & 1275 & 1089 & 4691 \\
\hline Hommes & $36 \%$ & $38 \%$ & $42 \%$ & $44 \%$ & $42 \%$ & $44 \%$ & $43 \%$ & $45 \%$ & $43 \%$ & $44 \%$ \\
\hline Femmes & $64 \%$ & $62 \%$ & $58 \%$ & $56 \%$ & $58 \%$ & $56 \%$ & $57 \%$ & $55 \%$ & $57 \%$ & $56 \%$ \\
\hline En couple & $85 \%$ & $84 \%$ & $90 \%$ & $75 \%$ & $79 \%$ & $77 \%$ & $73 \%$ & $85 \%$ & $72 \%$ & $77 \%$ \\
\hline
\end{tabular}




\begin{tabular}{|c|c|c|c|c|c|c|c|c|c|c|}
\hline Célibataires & $15 \%$ & $16 \%$ & $10 \%$ & $25 \%$ & $21 \%$ & $23 \%$ & $27 \%$ & $15 \%$ & $28 \%$ & $23 \%$ \\
\hline Mère en vie seulement & $66 \%$ & $64 \%$ & $67 \%$ & $61 \%$ & $63 \%$ & $58 \%$ & $57 \%$ & $61 \%$ & $62 \%$ & $60 \%$ \\
\hline Père en vie seulement & $16 \%$ & $17 \%$ & $14 \%$ & $18 \%$ & $17 \%$ & $12 \%$ & $13 \%$ & $10 \%$ & $12 \%$ & $12 \%$ \\
\hline Deux parents en vie & $17 \%$ & $19 \%$ & $19 \%$ & $21 \%$ & $20 \%$ & $30 \%$ & $30 \%$ & $29 \%$ & $25 \%$ & $29 \%$ \\
\hline $50-54$ ans & $6 \%$ & $5 \%$ & $27 \%$ & $34 \%$ & $27 \%$ & $48 \%$ & $27 \%$ & $36 \%$ & $23 \%$ & $35 \%$ \\
\hline $55-59$ ans & $51 \%$ & $42 \%$ & $45 \%$ & $38 \%$ & $41 \%$ & $30 \%$ & $37 \%$ & $37 \%$ & $44 \%$ & $36 \%$ \\
\hline $60-64$ ans & $43 \%$ & $53 \%$ & $28 \%$ & $28 \%$ & $32 \%$ & $22 \%$ & $36 \%$ & $27 \%$ & $33 \%$ & $28 \%$ \\
\hline Retraités & $21 \%$ & $8 \%$ & $8 \%$ & $22 \%$ & $18 \%$ & $7 \%$ & $7 \%$ & $7 \%$ & $24 \%$ & $11 \%$ \\
\hline En emploi & $56 \%$ & $85 \%$ & $51 \%$ & $60 \%$ & $61 \%$ & $72 \%$ & $85 \%$ & $58 \%$ & $59 \%$ & $67 \%$ \\
\hline Chômeurs actifs & $5 \%$ & $3 \%$ & $11 \%$ & $5 \%$ & $6 \%$ & $7 \%$ & $3 \%$ & $14 \%$ & $5 \%$ & $8 \%$ \\
\hline Autres, inactifs & $18 \%$ & $5 \%$ & $29 \%$ & $13 \%$ & $15 \%$ & $14 \%$ & $5 \%$ & $20 \%$ & $11 \%$ & $14 \%$ \\
\hline N'aide aucun parent & $80 \%$ & $71 \%$ & $83 \%$ & $83 \%$ & $81 \%$ & $76 \%$ & $68 \%$ & $77 \%$ & $78 \%$ & $75 \%$ \\
\hline Aide un parent & $20 \%$ & $29 \%$ & $17 \%$ & $17 \%$ & $19 \%$ & $24 \%$ & $32 \%$ & $23 \%$ & $22 \%$ & $25 \%$ \\
\hline N'aide aucun parent & $80 \%$ & $71 \%$ & $83 \%$ & $83 \%$ & $81 \%$ & $76 \%$ & $68 \%$ & $77 \%$ & $78 \%$ & $75 \%$ \\
\hline Aide la mère & $16 \%$ & $19 \%$ & $10 \%$ & $13 \%$ & $13 \%$ & $17 \%$ & $23 \%$ & $15 \%$ & $16 \%$ & $18 \%$ \\
\hline Aide le père & $1 \%$ & $5 \%$ & $4 \%$ & $2 \%$ & $3 \%$ & $3 \%$ & $5 \%$ & $4 \%$ & $2 \%$ & $3 \%$ \\
\hline Aide les deux parents & $3 \%$ & $5 \%$ & $3 \%$ & $2 \%$ & $3 \%$ & $4 \%$ & $4 \%$ & $4 \%$ & $4 \%$ & $4 \%$ \\
\hline Non corésidents & $96 \%$ & $100 \%$ & $89 \%$ & $98 \%$ & $96 \%$ & $97 \%$ & $99 \%$ & $88 \%$ & $97 \%$ & $95 \%$ \\
\hline Corésidents avec la mère & $3 \%$ & $0 \%$ & $7 \%$ & $2 \%$ & $3 \%$ & $2 \%$ & $1 \%$ & $9 \%$ & $3 \%$ & $4 \%$ \\
\hline Corésidents avec le père & $0 \%$ & $0 \%$ & $2 \%$ & $0 \%$ & $1 \%$ & $0 \%$ & $0 \%$ & $1 \%$ & $0 \%$ & $1 \%$ \\
\hline Corésidents avec les deux parents & $1 \%$ & $0 \%$ & $2 \%$ & $0 \%$ & $0 \%$ & $0 \%$ & $0 \%$ & $2 \%$ & $0 \%$ & $1 \%$ \\
\hline Santé excellente (mère) & $1 \%$ & $4 \%$ & $1 \%$ & $4 \%$ & $3 \%$ & $2 \%$ & $8 \%$ & $1 \%$ & $5 \%$ & $4 \%$ \\
\hline Santé très bonne (mère) & $3 \%$ & $10 \%$ & $6 \%$ & $6 \%$ & $6 \%$ & $6 \%$ & $13 \%$ & $5 \%$ & $6 \%$ & $7 \%$ \\
\hline Santé bonne (mère) & $21 \%$ & $21 \%$ & $31 \%$ & $32 \%$ & $30 \%$ & $33 \%$ & $21 \%$ & $32 \%$ & $32 \%$ & $31 \%$ \\
\hline Santé acceptable (mère) & $43 \%$ & $30 \%$ & $40 \%$ & $30 \%$ & $33 \%$ & $36 \%$ & $35 \%$ & $42 \%$ & $31 \%$ & $36 \%$ \\
\hline Santé médiocre (mère) & $32 \%$ & $33 \%$ & $22 \%$ & $26 \%$ & $27 \%$ & $20 \%$ & $22 \%$ & $20 \%$ & $24 \%$ & $21 \%$ \\
\hline Santé NR (mère) & $0 \%$ & $2 \%$ & $0 \%$ & $2 \%$ & $1 \%$ & $2 \%$ & $0 \%$ & $0 \%$ & $2 \%$ & $1 \%$ \\
\hline Total ayant la mère en vie & 155 & 177 & 255 & 956 & 1543 & 1365 & 682 & 1143 & 955 & 4145 \\
\hline Santé excellente (père) & $3 \%$ & $8 \%$ & $3 \%$ & $5 \%$ & $5 \%$ & $3 \%$ & $9 \%$ & $2 \%$ & $4 \%$ & $4 \%$ \\
\hline Santé très bonne (père) & $0 \%$ & $18 \%$ & $7 \%$ & $8 \%$ & $8 \%$ & $7 \%$ & $10 \%$ & $7 \%$ & $7 \%$ & $8 \%$ \\
\hline Santé bonne (père) & $21 \%$ & $28 \%$ & $32 \%$ & $27 \%$ & $27 \%$ & $30 \%$ & $26 \%$ & $37 \%$ & $33 \%$ & $32 \%$ \\
\hline Santé acceptable (père) & $39 \%$ & $20 \%$ & $42 \%$ & $31 \%$ & $32 \%$ & $36 \%$ & $32 \%$ & $37 \%$ & $27 \%$ & $34 \%$ \\
\hline Santé médiocre (père) & $35 \%$ & $24 \%$ & $16 \%$ & $25 \%$ & $25 \%$ & $20 \%$ & $21 \%$ & $16 \%$ & $28 \%$ & $21 \%$ \\
\hline
\end{tabular}




\begin{tabular}{|l|c|c|c|c|c|c|c|c|c|c|}
\hline Santé NR (père) & $2 \%$ & $3 \%$ & $0 \%$ & $4 \%$ & $3 \%$ & $3 \%$ & $2 \%$ & $0 \%$ & $2 \%$ & $2 \%$ \\
\hline Total ayant le père en vie & 62 & 76 & 98 & 450 & 686 & 646 & 336 & 498 & 410 & 1890 \\
\hline Aide quotidienne (mère) & $29 \%$ & $4 \%$ & $84 \%$ & $29 \%$ & $32 \%$ & $24 \%$ & $3 \%$ & $42 \%$ & $19 \%$ & $20 \%$ \\
\hline Aide hebdomadaire (mère) & $40 \%$ & $41 \%$ & $5 \%$ & $31 \%$ & $31 \%$ & $35 \%$ & $30 \%$ & $35 \%$ & $42 \%$ & $36 \%$ \\
\hline Aide mensuelle (mère) & $17 \%$ & $37 \%$ & $3 \%$ & $21 \%$ & $21 \%$ & $26 \%$ & $33 \%$ & $9 \%$ & $21 \%$ & $24 \%$ \\
\hline Aide moins souvent (mère) & $14 \%$ & $18 \%$ & $8 \%$ & $18 \%$ & $16 \%$ & $15 \%$ & $34 \%$ & $14 \%$ & $17 \%$ & $21 \%$ \\
\hline Aide NR (mère) & $0 \%$ & $0 \%$ & $0 \%$ & $0 \%$ & $0 \%$ & $0 \%$ & $0 \%$ & $0 \%$ & $0 \%$ & $0 \%$ \\
\hline Total aidant la mère & 35 & 51 & 38 & 174 & 298 & 288 & 212 & 110 & 189 & 799 \\
\hline Aide quotidienne (père) & $38 \%$ & $0 \%$ & $84 \%$ & $23 \%$ & $31 \%$ & $23 \%$ & $11 \%$ & $68 \%$ & $29 \%$ & $35 \%$ \\
\hline Aide hebdomadaire (père) & $50 \%$ & $32 \%$ & $5 \%$ & $33 \%$ & $29 \%$ & $40 \%$ & $32 \%$ & $18 \%$ & $35 \%$ & $31 \%$ \\
\hline Aide mensuelle (père) & $13 \%$ & $41 \%$ & $5 \%$ & $23 \%$ & $23 \%$ & $21 \%$ & $32 \%$ & $5 \%$ & $21 \%$ & $19 \%$ \\
\hline Aide moins souvent (père) & $0 \%$ & $27 \%$ & $5 \%$ & $21 \%$ & $18 \%$ & $17 \%$ & $24 \%$ & $7 \%$ & $15 \%$ & $15 \%$ \\
\hline Aide NR (père) & $0 \%$ & $0 \%$ & $0 \%$ & $0 \%$ & $0 \%$ & $0 \%$ & $0 \%$ & $1 \%$ & $0 \%$ & $0 \%$ \\
\hline Total aidant le père & 8 & 22 & 19 & 52 & 101 & 102 & 71 & 98 & 66 & 337 \\
\hline
\end{tabular}

\section{ANALYSE}

\section{I. PARENTS VIVANTS ET PARTICIPATION DANS LE MARCHÉ DU TRAVAIL}

Comme le montre le graphique I, la proportion d'individus âgés de 50-64 ans ayant au moins un parent en vie diminue à mesure que l'âge avance, et particulièrement la proportion de ces individus qui ont les deux parents en vie. L’espérance de vie des femmes étant plus élevée que celle des hommes, même si l'écart entre les deux tend à se réduire, les proportions d'individus ayant seulement leur mère en vie sont beaucoup plus importantes que celles des individus ayant seulement leur père en vie. Ainsi, à l'âge de 64 ans, les individus ayant au moins un parent vivant ne concernent quasi totalement que des individus ayant seulement leur mère en vie. Au-delà du fait évident que la proportion de la population ayant au moins un parent en vie diminue avec l'âge, nous pouvons constater qu'entre l'âge de 50 et 58 ans, la majorité des individus de l'échantillon dans l'ensemble des quatre pays ont au moins un parent en vie, tandis que ce taux s'élève encore à $30 \%$ à 64 ans. Dans la perspective de la volonté des 
pouvoirs publiques d'augmenter le taux d’activité sur le marché du travail des personnes âgées entre 55 et 64 ans, le fait que presque la moitié de la population étudiée ait au moins un parent en vie ne peut manquer de nous interroger quant au rapport entre des politiques destinées à prolonger la vie active et le rôle des aidants informels dans la prise en charges des personnes âgées.

Graphique I. Proportion d'individus ayant un ou deux parents en vie au moment de l'enquête en vague 5

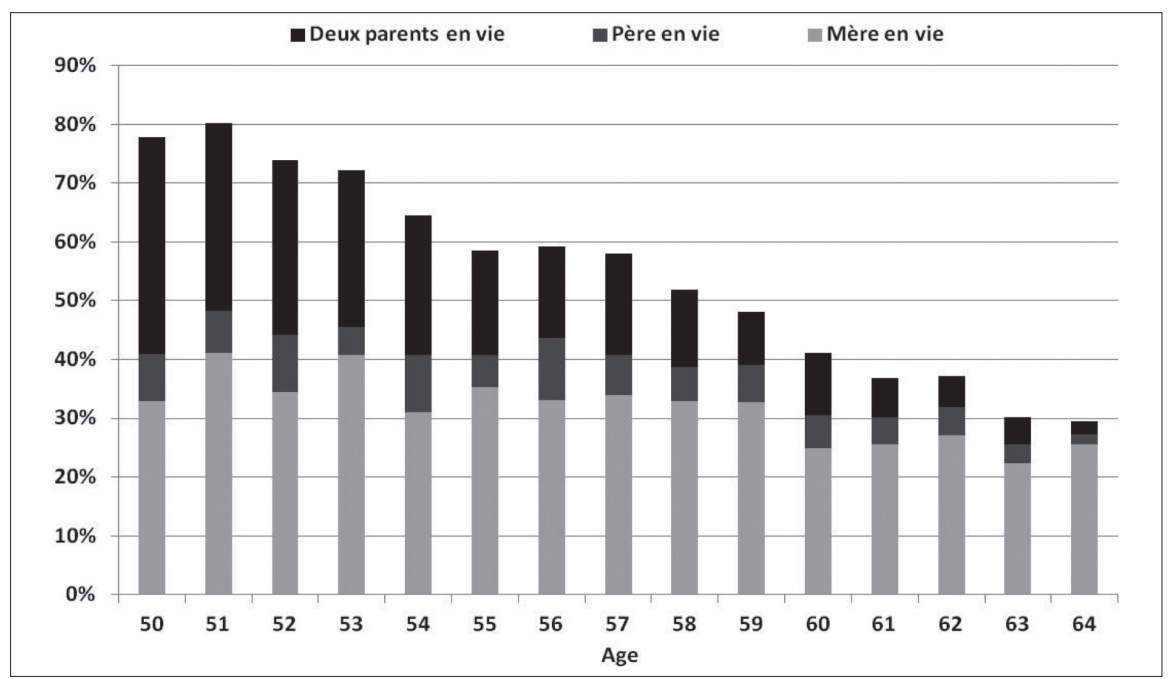

Note de lecture: parmi les quatre pays sélectionnés, les individus âgés de 51 ans sont 81 \% à avoir au moins un parent en vie, et $41 \%$ environ ont seulement leur mère en vie.

Champ: effectifs pondérés d'individus âgés entre 50 et 64 ans et ayant au moins un parent en vie en vague 5 de l'enquête «Share».

Source: enquête «Share», 469l individus en 2012.

Si la proportion d'individus ayant un ou deux parents en vie ne varie que faiblement entre les deux dernières vagues de l'enquête «Share», la situation des séniors sur le marché de l'emploi est plus sensible et a changé significativement. Quand on compare les taux d'emploi entre les vagues 4 (2010-20II) et 5 (2012-2013), on remarque ceux des hommes ont diminué dans tous les pays choisis à l'exception de l'Allemagne où le taux d'emploi des hommes augmente fortement au point de dépasser celui des femmes (cf. Tableau 2). À l'inverse, les taux d'emploi des femmes augmentent entre les deux vagues dans les pays sélectionnés, à l'exception 
de l'Espagne qui voit le taux d'emploi des femmes diminuer de plus de 3 points si l'on neutralise l'effet de la structure par âge. La baisse du taux d'emploi global en Espagne et en France entre les deux vagues résulte en grande majorité de la baisse des taux d'emploi des hommes. Nous pouvons aussi relever un axe NordSud sur cet indicateur, puisque la Suède dispose des taux d'emploi les plus élevés en comparaison de l’Espagne qui détient les taux d’emploi les plus faibles, tandis que la France et l'Allemagne se trouvent dans une situation intermédiaire.

Tableau 3. Taux d'emploi et taux d'emploi standardisés (en \%) selon le pays dans nos souséchantillons des vagues 4 et 5

\begin{tabular}{|c|c|c|c|c|}
\hline \multirow[b]{2}{*}{ PAYS } & \multirow[b]{2}{*}{ GENRE } & \multicolumn{3}{|c|}{ TAUX D'EMPLOI (EN\%) } \\
\hline & & VAGUE 4 & VAGUE 5 & $\begin{array}{c}\text { STANDARDISÉS } \\
\text { (vague } 4 \text { en pop. de réf.) }\end{array}$ \\
\hline \multirow{2}{*}{ Allemagne } & Hommes & 53,1 & 75,5 & 78,0 \\
\hline & Femmes & 69,7 & 70,2 & 70,5 \\
\hline \multirow{2}{*}{ Suède } & Hommes & 91,3 & 90,1 & 88,9 \\
\hline & Femmes & 80,2 & 85,1 & 84,9 \\
\hline \multirow{2}{*}{ Espagne } & Hommes & 70,5 & 62,6 & 55,4 \\
\hline & Femmes & 44,5 & 48,3 & 41,2 \\
\hline \multirow{2}{*}{ France } & Hommes & 67,8 & 64,5 & 62,1 \\
\hline & Femmes & 62,4 & 64,7 & 64,6 \\
\hline
\end{tabular}

Note de lecture: en Espagne, le taux d'emploi des hommes âgés entre 50 et 64 ans et ayant au moins un parent vivant était de $70,5 \%$ en vague 4 contre $62,6 \%$ en vague 5 . Si l'on neutralise l'effet de l'âge à l'aide d'une standardisation directe en appliquant à la vague 5 la structure par âge de cette population en vague 4, le taux d'emploi standardisé est plus faible que le taux d'emploi brut en vague 5.

Champ: effectifs pondérés d'individus âgés entre 50 et 64 ans et ayant au moins un parent en vie en vague 4 et en vague 5 de l'enquête «Share».

Source: enquête «Share», I 857 individus en 2010 et 4691 en 2012.

\subsection{DISPARITÉS ET ÉVOLUTION DES APPORTS D'AIDE INFORMELLE DES SÉNIORS}

La proportion d'enfants aidants n'est pas identique dans tous les pays d'Europe, nous relevons le gradient Nord-Sud inversé sur ce taux d'aide apportée à un parent âgé déjà repéré dans les vagues précédentes de l'enquête «Share» (Ogg et Renaut, 2006). Ainsi dans notre sous-échantillon en vague $5,34 \%$ des femmes suédoises et 31 \% 
des hommes suédois apportaient de l'aide à leur parent âgé alors qu'ils n'étaient respectivement que $28 \%$ de femmes et I3\% d'hommes aidants en Espagne, les pays d'Europe continentale étant dans une situation similaire voire intermédiaire (respectivement $22 \%$ et $16 \%$ en France, et $20 \%$ et $16 \%$ en Allemagne) ${ }^{3}$.

L'écart entre les hommes et les femmes en termes d’aide apportée à un parent âgé se distingue nettement. Ainsi, qu'elles aient ou non une activité, les femmes aident plus leur parent âgé que les hommes. Enfin, si l'on compare chaque situation par sexe et pays selon la situation d'emploi, on remarque quelques différences (cf. Tableau 4). Pour les hommes, alors que le taux d'aide est plus élevé pour ceux étant en emploi que ceux sans emploi en Allemagne (21 \% contre 16\%) et quasi équivalent en Suède et France (31 \% contre 30\% en Suède, $16 \%$ contre i7 $\%$ en France), la situation s'inverse en Espagne où le taux d’aide y est plus élevé pour ceux sans emploi (18\% contre 10\%). Pour les femmes, le taux d’aide est plus élevé pour celles en emploi en Suède (35\% contre $26 \%$ ) et dans une moindre mesure en Espagne (29\% contre $27 \%$ ), et inversement en France et en Allemagne où les femmes en emploi ont un taux d'aide similaire à celles sans emploi (respectivement 29\% contre $28 \%$ en France, et $28 \%$ contre $29 \%$ en Suède) tandis qu'en Allemagne il est le même pour les femmes en emploi et sans emploi (29\%). De plus, pour les quatre pays observés, les femmes en emploi ont un taux d’aide plus élevé que les hommes sans emploi alors qu'en comparaison à l'exception de la Suède, les hommes en emploi ont un taux d'aide plus faible que les femmes sans emploi. Cela peut être dû à la durée du travail, puisque les femmes forment la grande majorité des personnes travaillant à temps partiel qui est davantage compatible avec l'apport d'aide informelle qu'un travail à temps complet. La situation d'emploi vient donc accentuer les inégalités entre les hommes et les femmes face à l'apport d’aide à leur parent âgé.

3 Si l'on compare maintenant ces taux standardisés d'aide de cette vague 5 à ceux de la vague I (la structure par âge de la population en vague I étant la population de référence), nous observons que dans tous les cas les séniors sont moins nombreux en proportion à apporter de l'aide à un parent âgé quel que soit le sexe dans cette vague la plus récente, à l'exception de la France où ces taux augmentent pour les hommes et pour les femmes. La diminution de ces taux est la plus importante entre la vague I et la vague 4 dans la majorité des cas. 
Tableau 4. Taux d'aide apportée à un parent âgé en vague 5 selon le pays, le sexe et la situation d'emploi

\begin{tabular}{|l|c|c|c|c|c|c|c|}
\hline \multirow{2}{*}{} & \multicolumn{2}{|c|}{ HOMMES } & \multicolumn{2}{c|}{ FEMMES } & \multicolumn{2}{c|}{ ENSEMBLE } & \multirow{2}{*}{ TOTAL } \\
\cline { 2 - 7 } & $\begin{array}{c}\text { Sans } \\
\text { emploi }\end{array}$ & $\begin{array}{c}\text { En } \\
\text { emploi }\end{array}$ & $\begin{array}{c}\text { Sans } \\
\text { emploi }\end{array}$ & $\begin{array}{c}\text { En } \\
\text { emploi }\end{array}$ & $\begin{array}{c}\text { Sans } \\
\text { emploi }\end{array}$ & $\begin{array}{c}\text { En } \\
\text { emploi }\end{array}$ & TO \\
\hline Allemagne & $16 \%$ & $21 \%$ & $29 \%$ & $28 \%$ & $23 \%$ & $25 \%$ & $24 \%$ \\
\hline Suède & $30 \%$ & $31 \%$ & $26 \%$ & $35 \%$ & $28 \%$ & $33 \%$ & $32 \%$ \\
\hline Espagne & $18 \%$ & $10 \%$ & $27 \%$ & $29 \%$ & $23 \%$ & $18 \%$ & $21 \%$ \\
\hline France & $17 \%$ & $16 \%$ & $28 \%$ & $29 \%$ & $23 \%$ & $23 \%$ & $23 \%$ \\
\hline Total aidants & 124 & 299 & 251 & 486 & 375 & 785 & 1160 \\
\hline Total & 613 & 1453 & 913 & 1712 & 1526 & 3165 & 4691 \\
\hline
\end{tabular}

Note de lecture: en Suède, $26 \%$ des femmes sans emploi âgées de 50-64 ans et ayant au moins un parent vivant apportaient de l'aide à un parent en vague 5 , contre $35 \%$ de celles en emploi.

Champ: effectifs pondérés d'individus âgés entre 50 et 64 ans et ayant au moins un parent en vie en vague 5 de l'enquête «Share».

Source: enquête «Share», 4691 individus en 2012.

Comme nous l'avons constaté, il n'est pas possible d'évaluer l'aide informelle à un parent âgé sans prendre en compte les différences de modes de vie et notamment la corésidence intergénérationnelle. De fait, il existe également un gradient Nord-sud inversé concernant la corésidence entre les enfants âgés de 50-64 ans et leurs parents (cf. Tableau 4). En Suède la proportion de corésidents est très faible voire quasi inexistante alors qu'en Espagne cette proportion est très forte, tandis qu'en France et en Allemagne la situation est intermédiaire. Ces différences peuvent être expliquées en partie par les politiques nationales relatives à la dépendance, puisque dans les pays du Sud de l'Europe comme en Espagne elles favorisent le maintien à domicile et le soutien familial contrairement à la Suède où les politiques publiques favorisent davantage la prise en charge en institution. Lorsque l'on se concentre sur la comparaison parmi les aidants entre les deux vagues (cf. Tableau 5), on note une stabilité de la proportion de corésidents en Allemagne, une légère hausse en Suède et en France, mais une forte baisse en Espagne où la proportion reste tout de même élevée. Ces proportions révèlent les modèles d’aide informelle donnée aux parents âgés dans les pays du Nord, où la corésidence entre les séniors et leurs parents 
est quasi inexistante, et dans les pays du Sud où l'aide des séniors à leurs parents passe dans la grande majorité des cas par la corésidence.

Tableau 5. Taux de séniors non corésidents et corésidents avec leurs parents parmi les aidants en vague 4 et 5

\begin{tabular}{|l|c|c|c|c|}
\hline \multirow{2}{*}{ PAYS } & \multicolumn{2}{|c|}{ VAGUE 4 } & \multicolumn{2}{c|}{ VAGUE 5 } \\
\cline { 2 - 5 } & $\begin{array}{c}\text { Non } \\
\text { corésidents }\end{array}$ & Corésidents & $\begin{array}{c}\text { Non } \\
\text { corésidents }\end{array}$ & Corésidents \\
\hline Allemagne & $88 \%$ & $12 \%$ & $89 \%$ & $11 \%$ \\
\hline Suède & $99 \%$ & $1 \%$ & $97 \%$ & $3 \%$ \\
\hline Espagne & $43 \%$ & $57 \%$ & $56 \%$ & $44 \%$ \\
\hline France & $89 \%$ & $11 \%$ & $85 \%$ & $15 \%$ \\
\hline Total & 1789 & 68 & 4445 & 246 \\
\hline
\end{tabular}

Note de lecture: parmi les individus de nos sous-échantillons considérés comme aidants, $12 \%$ corésidaient avec leurs parents en vague 4 en Allemagne contre $57 \%$ en Espagne.

Champ: effectifs pondérés, en vague 4 et en vague 5 de l'enquête "Share», d'individus âgés entre 50 et 64 ans ayant au moins un parent en vie et étant considérés comme aidants.

Source: enquête «Share, 1857 individus en 2010 et 4691 individus en 2012.

Nous avons vu précédemment que les taux d’aide prennent à contre-pied le traditionnel gradient Nord-Sud selon lequel l'aide apportée des séniors à leurs parents âgés serait plus fréquente dans les pays d’Europe du Nord que ceux du Sud. Cependant, cet indicateur ne tient pas compte de l'intensité de l'aide apportée qui, comme les vagues précédentes de l’enquête «Share» ont également montré, est très variable d'un pays à l'autre. Il faut noter que l'intensité de l'aide apportée dans le questionnaire de l'enquête «Share» n'est mesurable que pour les enfants aidants non corésidents avec leurs parents âgés. La prise en compte de la fréquence de l'aide apportée par les enfants aidants (non corésidents) à leurs parents âgés fait ressurgir le traditionnel gradient Nord-Sud, où cette fréquence serait plus intensive dans les pays d'Europe du Sud et dans ceux du Nord, qui vient également être amplifié par la prise en compte de notre hypothèse des enfants corésidents comme aidants quotidiens. La Suède se distingue par une proportion très faible (moins de $5 \%$ ) d'enfants aidant quotidiennement, tandis qu'à l'inverse l'Espagne connaît des proportions d'en- 
fants aidant quotidiennement malgré une baisse significative entre les deux vagues. La France et l'Allemagne, elles, conservent leurs places d'intermédiaires en comparaison avec la Suède et l'Espagne. Ici encore, le modèle des politiques publiques relatives à la dépendance jouent un rôle important puisqu'en Suède les personnes âgées dépendantes sont davantage prises en charge en institution alors qu’en Espagne, les pouvoirs publics délèguent la prise en charge aux familles.

\subsection{LES DÉTERMINANTS DE LA PROBABILITÉ D'ÊTRE EN EMPLOI ET D'APPORT EN AIDE INFORMELLE DES SÉNIORS}

\section{Encadré 2. Méthode des rapports de cotes}

La régression logistique est une généralisation d'un coefficient servant à évaluer la corrélation de deux variables qualitatives: le rapport des cotes ou odds-ratio.

On appelle cote du succès le rapport:

$$
\exp (\theta)=\frac{\pi}{1-\pi}
$$

où $\pi$ est la probabilité de succès.

L'objectif est donc de calculer un coefficient qui permette de tester la probabilité de succès d'une variable par rapport à une autre.

Dans notre exemple, les rapports de cotes vont nous permettre de tester la variable d'apport d'aide à un parent et nous indiquer si les individus qui aident un parent ont plus ou moins de chance d'être en emploi par rapport à ceux qui n'en aident aucun.

Nous souhaitons analyser cette fois la probabilité d'être en emploi par pays en vague 5 à l'aide de rapports de cotes, également appelés odds-ratio, et d'une régression logistique. Ainsi, que ce soit en Suède, Allemagne ou Espagne, les femmes ont beaucoup moins de chances d'être en emploi que les hommes, plus nettement en Espagne qu'en Suède, alors que pour la France le résultat n’est pas significatif. Par tranche d'âge, on note que les 55-59 ans ont I,5 fois plus de chances d'être en emploi que les 50-54 ans en Allemagne, et I,2 fois plus en France. À l'inverse les 55-59 ans ont moins de chances d'être en emploi que les 
50-54 ans en Espagne, et également légèrement moins en Suède. La situation conjugale a aussi son importance puisqu'en Allemagne et en Suède, les célibataires ont moins de chances d'être en emploi que les personnes en couple, alors qu'à l'inverse en Espagne et dans une moindre mesure en France, les personnes célibataires ont plus de chances d'être en emploi que celles en couple.

Concernant l'aide apportée ou non à un parent âgé, les individus qui n’apportent pas d'aide ont beaucoup moins de chance d'être en emploi que ceux qui aident au moins un de leur parent en Suède et en Espagne, tandis qu'en France et légèrement en Allemagne, les individus n'aidant aucun parent ont plus de chances d'être en emploi que ceux apportant une aide à un parent âgé. Pour la corésidence, les individus ne résidant pas avec leurs parents ont légèrement plus de chances d'être en emploi que ceux qui sont corésidents, quel que soit le pays, la Suède exceptée où le résultat n’est pas significatif.

Dans ce modèle nous avons également intégré l’intensité de l’aide apportée aux parents âgés agrégée en trois catégories : l’aide régulière (aide quotidienne ou hebdomadaire, corésidence compris), l'aide occasionnelle (aide mensuelle ou moins souvent), et aucune aide. Quand l'aide est apportée à la mère, les individus aidant occasionnellement et ceux n'aidant pas ont plus de chances d'être en emploi que ceux qui aident régulièrement leur mère. Pour l'aide apportée au père, les aidants occasionnels ont beaucoup plus de chances d'être en emploi en Suède (2,I fois plus) et en France (2,5 fois plus) que ceux qui aident régulièrement. À l'inverse, ils ont moins de chances d'être en emploi que ceux qui aident régulièrement leur père en Allemagne et en Espagne. Les individus n'aidant pas leur père ont plus de chance d'être en emploi que les aidants réguliers quel que soit le pays, à l'exception de l’Allemagne. 


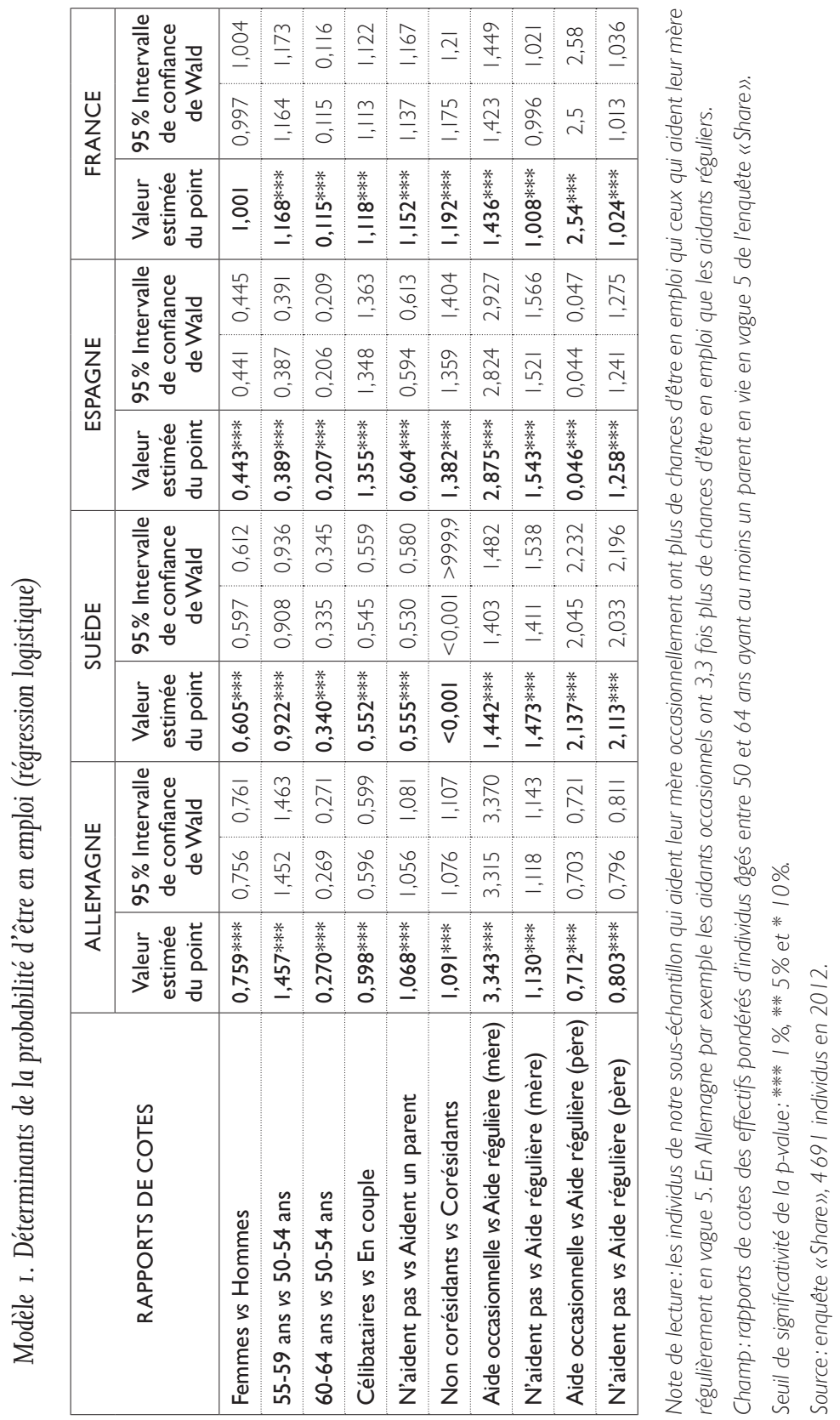


Dans un deuxième modèle, nous souhaitons connaître les déterminants de la probabilité d’apport d'aide à la mère sans tenir compte de l'intensité de cette aide. Relativement au sexe, les femmes ont plus de chances d'apporter de l'aide à leur mère que les hommes, quel que soit le pays, ce rapport étant très fort pour les espagnoles (3,9 fois plus) et plus faible pour les suédoises (I,3 fois plus). Les tranches d'âge les plus élevées correspondent quasi exclusivement à celles ayant une probabilité plus élevée d'apporter de l'aide à la mère, à l'exception des allemandes âgées de 55-59 ans qui ont légèrement moins de chance d’apporter de l'aide à leur mère que leurs compatriotes âgées de 50-54 ans. Le statut marital a également son importance puisque les célibataires ont plus de chances d’apporter de l'aide à leur mère que les individus en couple, plus particulièrement en France (2,4 fois plus) et en Espagne (I,8 fois plus).

L’activité a également une incidence sur la probabilité d'apporter de l'aide à sa mère âgée. Ainsi, cette probabilité est plus élevée pour les personnes au foyer que pour les chômeurs, et plus élevée pour les retraités que pour les chômeurs quel que soit le pays. Les personnes invalides ont légèrement plus de chances d'aider leur mère que les chômeurs actifs en Suède et en France, et moins de chances en Allemagne et en Espagne. Enfin, les salariés ont plus de chances d'apporter de l'aide à leur mère âgée que les chômeurs quel que soit le pays, à l'exception de la France où ce rapport de cote est légèrement inversé.

La perception de la santé de la mère a également une forte incidence sur la probabilité d'apporter de l'aide à celle-ci. Ainsi, plus les individus jugent la santé de leur mère de manière positive moins ils ont de chances d'apporter de l'aide à celle-ci, à l'exception des suédois jugeant la santé de leur mère bonne qui ont plus de chance d’apporter de l’aide à celle-ci que ceux la jugeant médiocre. 


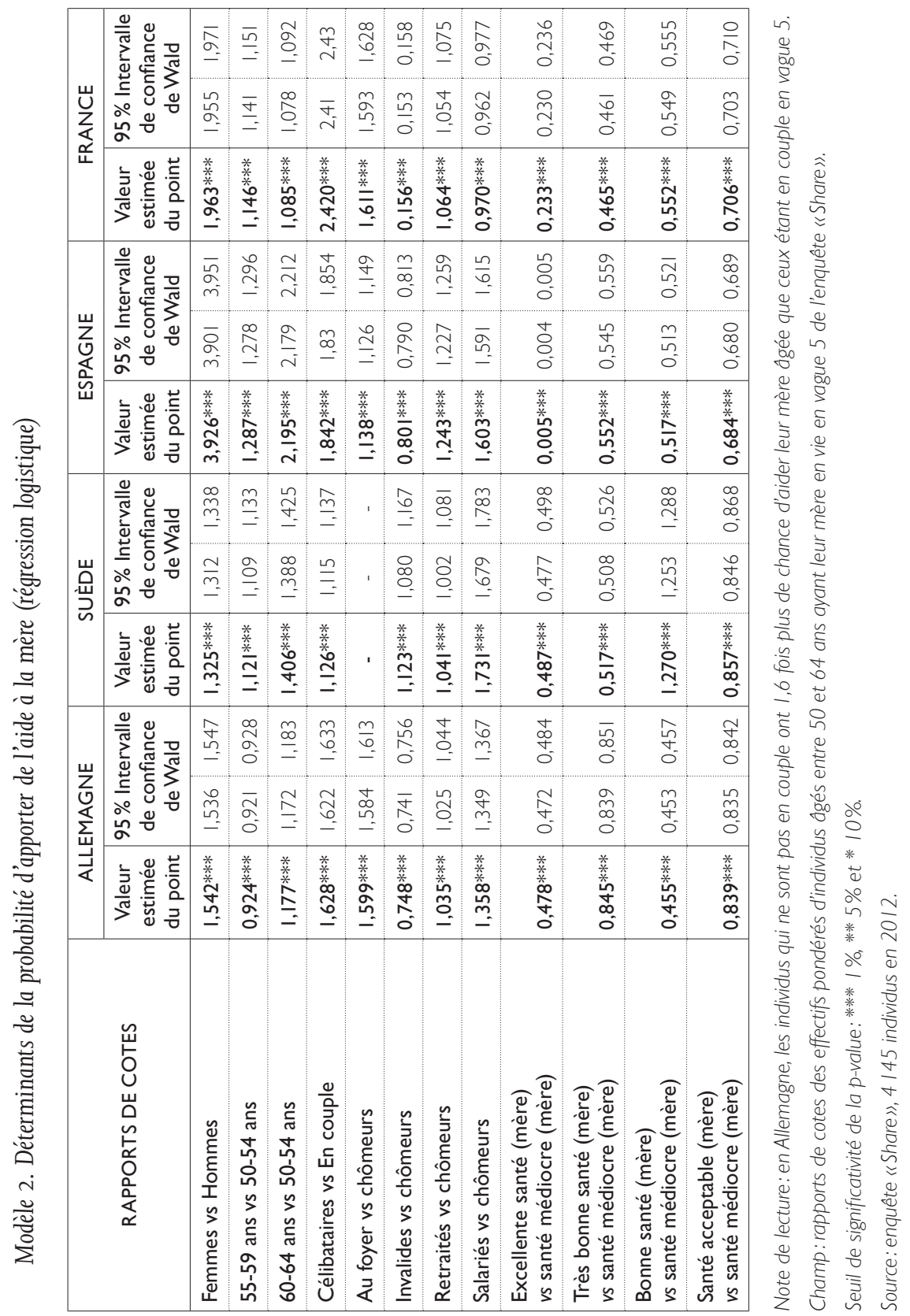


Enfin, dans un troisième modèle, nous souhaitons connaître les déterminants de la probabilité d’apport d'aide au père sans tenir compte de l'intensité de cette aide. Tout d'abord, en Allemagne et encore davantage en France, les femmes ont plus de chances d’apporter de l'aide à leur père que les hommes, à l'inverse de l'Espagne et de la Suède où ce sont les hommes qui ont davantage de probabilités d'apporter de l'aide à leur père que les femmes. Pour l'âge, la France fait figure d'exception puisque les 55-59 ans ont plus de chances d'apporter de l'aide à leur père que les personnes âgées entre 50 et 54 ans, tandis que les 60-64 ans en ont moins que ces derniers. À l'inverse en Espagne, les individus appartenant à la tranche des 55-59 ans ont moins de probabilités d’apporter de l'aide à leur père par rapport aux plus jeunes, tandis que les individus appartenant à la tranche d'âge des 60-64 ans ont 3,3 fois plus de chances d'apporter de l'aide à leur père que les 50-54 ans. En Allemagne et en Suède, à l'exception des suédois âgés de 60-64 ans qui ont légèrement plus de chances d’apporter de l'aide à leur père que les 50-54 ans, les rapports de cotes sont quasiment dans une situation d'équilibre telle que les chances d’apporter de l'aide au père sont identiques entre les tranches d'âge. Le statut marital a également son importance puisque les célibataires ont beaucoup plus de probabilités d'apporter de l'aide à leur père que les individus en couple, cela étant d’autant plus vrai au Sud de l’Europe et plus nuancé en Suède.

L'activité a une incidence sur la probabilité d’apporter de l'aide à son père âgé. Ainsi, à l'inverse de l'Allemagne, cette probabilité est plus élevée en Espagne et en France pour les personnes au foyer que pour les chômeurs en recherche active d'emploi. Les personnes en invalidité ont moins de chances d'apporter de l'aide à leur père âgé que les chômeurs actifs, quel que soit le pays. À l'exception de la France, les retraités et les salariés ont moins de chances d’apporter de l'aide à leur père que les chômeurs actifs. Cependant en France, le rapport de cotes entre les salariés et les chômeurs actifs montre une probabilité quasi identique d’apport d’aide au père. Ces probabilités peuvent être également le reflet de la conjoncture économique et des politiques relatives à la dépendance. On peut aider ses parents plus facilement en étant sans emploi tout en cherchant à travailler, et cela est d'autant plus fort en Espagne voire en France où le 
taux de chômage est élevé pour les séniors en âge d'être encore actifs et où les pouvoirs publics favorisent un maintien à domicile contrairement à la Suède.

La perception de la santé du père a également une forte incidence sur la probabilité d'apporter de l'aide à celui-ci. À l'exception de la Suède, les individus jugeant la santé de leur père excellente ont beaucoup moins de chance de lui apporter de l'aide que ceux jugeant l'état de santé de leur père médiocre. Il est en de même pour ceux jugeant l'état de santé de leur père très bon par rapport à ceux la jugeant médiocre, à l'exception de l'Allemagne où le rapport de cote nous indique une probabilité quasi identique entre les deux situations. Quel que soit le pays, les individus jugeant l'état de santé de leur père bon ont moins de chance d'apporter de l'aide à celui-ci que ceux jugeant l'état de santé du père médiocre. Enfin, les rapports de cotes entre ceux jugeant l'état de santé de leur père acceptable et ceux la jugeant médiocre nous indique une probabilité quasi identique d'apporter de l'aide à ce dernier, à l'exception de l'Espagne où les individus jugeant l'état de santé du père acceptable ont beaucoup moins de chances d’apporter de l’aide à celui-ci que ceux l’ayant jugé médiocre. 


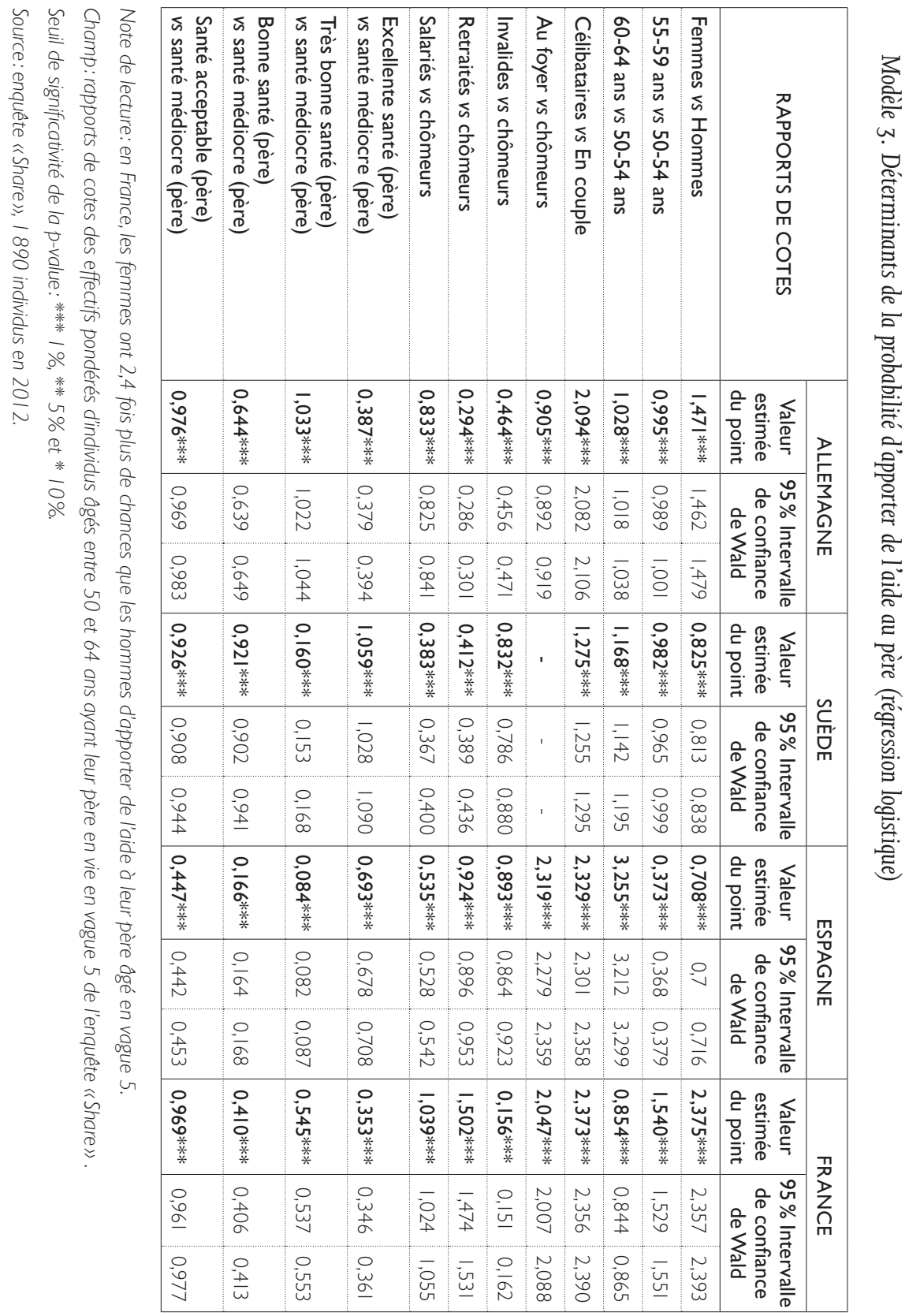




\section{CONCLUSION}

Les résultats de cette analyse permettent de souligner les différences d'évolution dans les quatre pays choisis concernant le lien entre l'emploi et l'apport d'aide informelle à un parent âgé, et notamment dans une période de crise voire d’après crise économique. Cependant, il faut tenir compte du fait que nos résultats sont dépendants de notre hypothèse de départ consistant à considérer tous les corésidents comme aidants quotidiens. Le taux d'emploi standardisé suit un gradient Nord-Sud. En effet, cet indicateur est le plus élevé en Suède et le plus bas en Espagne alors que la France et l'Allemagne sont dans une situation dite intermédiaire. Ce taux augmente également entre les deux vagues observées en Allemagne et en Suède, alors qu'il diminue en France et en Espagne ce qui vient creuser l'écart déjà constaté en vague 4.

La proportion d'enfants aidants (corésidents compris) sans prise en compte de l'intensité de l'aide apportée par ces derniers, est plus élevée en Suède que dans les autres pays, l'Espagne se distinguant par la proportion la plus faible parmi les quatre pays choisis alors que l'Allemagne et la France se trouvent l'entre deux. Si l'on regarde cette situation par genre selon la situation d'emploi, les femmes en emploi apportent plus d'aide que les hommes sans emploi alors qu'à l'inverse les hommes en emploi apportent moins d'aide que les femmes sans emploi. La situation vis-à-vis de l'emploi vient donc conforter le postulat de départ que les femmes restaient principalement les pourvoyeuses d'aides informelles. Si l'on tient compte cette fois de l'intensité de l'aide apporté aux parents âgés (corésidents compris), nous relevons que cet indicateur suit le gradient Nord-Sud. En effet, cet indicateur est le plus élevé dans les pays du Sud de l'Europe tels que l'Espagne, le plus faible dans les pays du Nord de l'Europe tels que la Suède, et dans une situation intermédiaire dans les pays continentaux tels que la France et l'Allemagne. L'écart dans l'intensité de l'aide apportée aux parents âgés entre les pays du Sud de l'Europe et ceux du Nord vient se creuser par la corésidence intergénérationnelle qui est courante en Espagne par exemple alors qu'elle est quasi inexistante en Suède. 
Tous ces résultats se confirment dans les rapports de cotes des différents modèles de régressions logistiques réalisés. On y relève également l'importance de l'intensité de l'aide donnée sur la probabilité d'être en emploi. Cependant, ce résultat demande une attention particulière dans son analyse puis que le lien entre les deux variables n'est pas un simple lien de cause à effet mais une double relation causale. Enfin les deux derniers modèles de régression logistique, portant sur les déterminants de l'apport d’aide à un parent âgé, nous indiquent l'importance de l'activité du pourvoyeur d'aide, quand bien même s'il s'agit ici aussi d'une double relation causale puisque l'aide apportée peut influer sur la situation à l'emploi et inversement. Pour finir, on relève également dans ces deux derniers modèles l'importance de la perception de la santé du parent : en général, plus l'état de santé de celui-ci est perçu négativement par l'enfant, plus la probabilité que ce dernier apporte de l'aide en temps à son parent est élevée.

À l'avenir, les parents âgés vivront plus longtemps sans pour autant être tous en bonne santé tandis que leurs enfants potentiellement aidants verront leur départ à la retraite de plus en plus tardif. Aussi, avec l'allongement de la durée d'activité et les gains d'espérance de vie, l'articulation entre l'aide informelle apportée à un proche et la vie professionnelle risque de se complexifier, mettant sous pression les systèmes de protection sociale en ce qui concerne les aides formelles. Le défi des prochaines décennies est donc non seulement le financement des systèmes de protection sociale mais aussi leur articulation avec les modèles d'aides formelles et informelles mis en place afin de répondre à une demande d’aide et de soins chez les personnes âgées de plus en plus soutenue.

\section{BIBLIOGRAPHIE}

ASSEMBLÉE NATIONALE, COMMISSION DES AFFAIRES EUROPÉENNES, 20II, Rapport d'information n. 3667, «La prise en charge de la dépendance en Europe»: <http://www.assemblee-nationale.fr//3/europe/rap-info/i3667.asp>.

ATTIAS-DONFUT C. et OGG J., 2009, «Évolution des transferts intergénérationnels: vers un modèle européen?», Retraite et Société, 58, p. II-29. 
BOLIN K., LINDGREN B. et LUNDBORG P., 2008, «Your Next of Kin or Your Own Career? Caring and Working Among The 50+ of Europe», Journal of Health Economics, 27, p. 718-738.

BONSANG E., 2009, «Does Informal Care from Children to Their Elderly Parents Substitute for Formal Care in Europe?», Journal of Health Economics, 28, p. 143-154.

COMMISSION DES COMPTES DE LA SÉCURITÉ SOCIALE, 20I0, Rapport 12-3, «Âges légaux et âges effectifs de cessation d'activité: comparaisons internationales»: <http://www.securite-sociale.fr/IMG/pdf/ccss201006_fic-12-3.pdf>.

DAVIN B., PARAPONARIS A. et VERGER P., 2009, «Entre famille et marché: déterminants et coûts monétaires de l'aide formelle et informelle reçue par les personnes âgées en domicile ordinaire», Management \& Avenir, 26-6, p. 190-204.

ESPING-ANDERSEN G., 1990, The Three Worlds of Welfare Capitalism, Princeton University Press, Princeton.

FONTAINE R., 2009, «Aider un parent âgé se fait-il au détriment de l'emploi?», Retraite et Société, 58, p. 31-6I.

-, GRAMAIN A. et WITTWER J., 2007, «Les configurations d'aide familiales mobilisées autour des personnes âgées dépendantes en Europe», Economie et Statistique, 403-404, p.97-115.

GILL T. M., GAHBAUER E. A., HAN L. et ALLORE H. G., 20I0, «Trajectories of Disability in the Last Year of Life», The New England Journal of Medicine, 362-13, p. $|173-1| 80$.

GUNER N., KAYA E. et SANCHEZ-MARCOS V., 2014, «Gender gaps in Spain: policies and outcomes over the last three decades», SERIEs, I-5, p. 6I-103.

HANK K., 2007, «Proximity and Contacts Between Older Parents and Their Children: A European Comparison», Journal of Marriage and Family, 69, p. 157-173.

KEMPER P., 1992, «The Use of Formal and Informal Home Care by The Disabled Elderly », Health Services Research, 27-4, p.421-45I.

KOHLI M., KÜNEMUND K. et LÜDICKE J., 2005, «Family Structure, Proximity and Contact», in SHARE First Results Book, Chapter 4.I. 
LE BIHAN-YOUINOU B. et MARTIN C., 2006, «Travailler et prendre soin d'un parent âgé dépendant», Travail, Genre et Sociétés, 16, p.77-96.

LITWIN H. et ATTIAS-DONFUT C., 2009, «The Inter-Relationship Between Formal and Informal Care: A Study in France and Israel», Ageing \& Society, 29, p.7I-91.

MINNI C., 2015, «Emploi et chômage des 55-64 ans en 20I3: tassement du taux d'activité, forte hausse du taux de chômage», Dares Analyses, 12.

OGG J. et RENAUT S., 2006, «The Support of Parents in Old Age by Those Born During 1945-1954: A European Perspective», Ageing \& Society, 26-5, p. 723-743.

ROE B., WHATTAM M., YOUNG H. et DIMOND M., 200I, «Elders' Perceptions of Formal and Informal Care: Aspects of Getting and Receiving Help for Their Activities of Daily Living», Journal of Clinical Nursing, 10-3, p. 398-405.

ULMANEN P. et SZEBEHELY M., 20I5, «From the State to the Family or to the Market? Consequences of Reduced Residential Eldercare in Sweden », International Journal of Social Welfare, 24-I, p. 81-92. 